\title{
High-density seedling expression system for the production of bioactive human cardiotrophin-1, a potential therapeutic cytokine, in transgenic tobacco chloroplasts
}

Imma Farrann ${ }^{1}$, Francisco Río-Manterola', María Íñiguez², Sonia Gárate², Jesús Prieto ${ }^{2}$ and Angel M. Mingo-Castel ${ }^{1, *}$

${ }^{1}$ Instituto de Agrobiotecnología, UPNA-CSIC-Gobierno de Navarra, Campus Arrosadía, 31006 Pamplona, Spain

${ }^{2}$ Área de Terapia Génica y Hepatología, CIMA, 31008 Pamplona, Spain

Received 21 December 2007; revised 27 February 2008; accepted 5 March 2008 *Correspondence (fax 34-948-232191; e-mail angelma@unavarra.es)
Keywords: cardiotrophin-1, chloroplast, expression regulatory elements, molecular farming, seedling expression system, tobacco.

\begin{abstract}
Summary
Histidine-tagged human cardiotrophin-1 (hCT-1), a recently discovered cytokine with excellent therapeutic potential, was expressed in tobacco chloroplasts under the transcriptional and translational control of two different promoters ( $r r n$ and $p s b A$ ) and 5'-untranslated regions (5'-UTRs) (psbA and phage T7 gene 10). The psbA 5'-UTR promotes recombinant hCT-1 (rhCT-1) accumulation in chloroplasts at higher levels (eight-fold) than those obtained for the phage 77 gene $105^{\prime}$-UTR, regardless of the promoter used, indicating that the correct choice of translational control element is most important for protein production in chloroplasts. The maximum level of rhCT-1 achieved was $1.14 \mathrm{mg} / \mathrm{g}$ fresh weight (equivalent to $5 \%$ of total soluble protein) with the $p s b A$ promoter and $5^{\prime}$-UTR in young leaves harvested after $32 \mathrm{~h}$ of continuous light, although the bioactivity was significantly lower $(\sim 35 \%)$ than that of commercial hCT-1. However, harvesting in the dark or after $12 \mathrm{~h}$ of light did not result in a significant decrease in the bioactivity of rhCT-1, suggesting that $32 \mathrm{~h}$ of over-lighting affects the biological activity of rhCT-1. Because high levels of rhCT-1 accumulation took place mainly in young leaves, it is proposed that seedlings should be used in a 'closed system' unit, yielding up to $3.2 \mathrm{~kg}$ per year of rhCT-1. This amount would be sufficient to meet the estimated annual worldwide needs of hCT-1 for liver transplantation surgery in a cost-effective manner. Furthermore, our strategy is an environmentally friendly method for the production of plant-based biopharmaceuticals.
\end{abstract}

\section{Introduction}

New discoveries involving therapeutic proteins offer exciting possibilities for the treatment of human pathologies. Several challenges still exist in the production of these therapeutic proteins. They must be obtained on a cost-effective basis in sufficient amounts to meet current growing needs. Plantmade pharmaceuticals are becoming a major commercial development in biotechnology (Ma et al., 2005). They offer significant advantages over other production systems, such as low production costs, easy scale-up, absence of human pathogens and convenient storage and processing. Although the plant nuclear genome has been used to express human therapeutic proteins for more than a decade (Fischer et al., 2004), expression levels have been disappointingly low, usually far lower than $1 \%$ of total soluble protein (TSP), a yield that is estimated by industry to be sufficient for the production of biopharmaceuticals in a cost-effective manner (Farran et al., 2002).

The chloroplast transgenic approach has been developed as an alternative to nuclear expression (Daniell, 2006; Bock, 2007). The accumulation of exceptionally large quantities of 
foreign proteins (De Cosa et al., 2001; Molina et al., 2004) has been achieved partly as a result of high transgene polyploidy (up to 20000 copies/cell) (Maliga, 2004). Human therapeutic proteins, such as somatotropin (Staub et al., 2000), serum albumin (Fernández-San Millán et al., 2003), various interferon types (Leelavathi and Reddy, 2003; Arlen et al., 2007) and proinsulin (Ruhlman et al., 2007), have been produced at high levels (6\%-20\% of TSP). However, other attempts have failed (Magee et al., 2004; Wirth et al., 2006), indicating that post-transcriptional mechanisms are likely to be involved. Chloroplast-derived therapeutic proteins have also shown correct folding, disulphide bond formation and functionality (Daniell, 2006). Other advantages include a much more stable and uniform expression among transgenic lines, the absence of epigenetic effects and gene silencing, the ability to simultaneously express multiple transgenes by linking them together in operons (Quesada-Vargas et al., 2005), an increase in transgene containment as a result of the maternal inheritance of plastids in most crop plants (Hagemann, 2004), and the expression of foreign proteins lacking methionine as the N-terminal amino acid (FernándezSan Millán et al., 2007).

Cardiotrophin-1 (CT-1) is a member of the interleukin- 6 family, which is structurally and biologically related to cytokines. It was first identified by its ability to induce hypertrophic response in cardiac myocytes (Pennica et al., 1995). CT-1 induces its biological effects through the shared signalling subunit gp130 (Pennica et al., 1996a), and stimulates the Janus kinase/signal transducers and activators of transcription (JAK/STAT) pathway to transmit hypertrophic and cytoprotective signals (Robledo et al., 1997). It has been reported that recombinant CT-1 (rCT-1) may defend cardiac cells against hypoxic damage (Brar et al., 2001), hepatocytes against acute liver injury (Bustos et al., 2003) and neurones against oxidative injury (Wen et al., 2005). Recently, CT-1 has been defined as a natural defence of the liver against apoptosis (Marques et al., 2007) and ischaemia-reperfusion injury, and is a key mediator of the protective effect induced by ischaemic preconditioning (Iñiguez et al., 2006). From all these findings, it is conceivable that this cytokine may offer a high therapeutic potential. Other valuable therapeutic uses of CT-1 are in the treatment of spinal muscular atrophy (Lesbordes et al., 2003) and in surgery for liver cancer or transplantation (Bustos et al., 2003). Unfortunately, the only human rCT-1 (rhCT-1) commercially available is produced in Escherichia coli at a cost of about $\$ 5000 / \mathrm{mg}$. As a consequence, no in vivo assays have been performed with the high-cost rhCT-1 to date. To our knowledge, no specific literature concerning recombinant expression systems for
hCT-1 protein has been reported. Pennica et al. (1996b) referred to the production of hCT-1 in human 293 cells as part of their experimental procedure, but no production data were given. Alternative strategies are therefore required to reduce costs to facilitate potential therapeutic application.

In this study, the production of rhCT-1 in tobacco chloroplasts is pursued. The hCT-1 protein contains 201 amino acids, two cysteine residues (no experimental evidence of disulphide bridge linkage has been reported) and lacks potential N-linked glycosylation sites (Pennica et al., 1996b). It is therefore a good candidate for chloroplast expression. The hCT-1 was generated as a recombinant protein with $\mathrm{N}$-terminal fusion of a histidine (His) tag and enterokinase cleavage site. The optimization of the yield of recombinant protein in transplastomic plants is a key factor for cost-effective production. In this context, the effectiveness of different 5'-regulatory sequences, leaf age and growing conditions on the expression level and bioactivity of the protein were analysed. High yields of rhCT-1 were achieved by harvesting young tobacco leaves grown in a confined environment. For the first time, it was found that the hCT-1 bioactivity level produced in chloroplasts can be down-regulated by over-lighting.

\section{Results and discussion}

\section{Chimeric rhCT-1 genes and vector construction}

To achieve high levels of rhCT-1 protein accumulation in plastids, two chimeric genes were initially tested, each of which carried the $3^{\prime}$-mRNA stability elements from the chloroplast ribosome small subunit 16 gene (Trps16) (Staub and Maliga, 1994). To optimize hCT-1 protein accumulation in transgenic chloroplasts, different 5'-regulatory sequences were used. In the plasmid pL3-PrrnG10L-CT1 (Figure 1a), the rhct1 gene was expressed from the plastid constitutive promoter of the rRNA operon (Prrn) fused to the leader sequence of the phage T7 gene 10 (G10L). High levels of foreign protein expression in chloroplasts (7\%-25\% of TSP) have been obtained for different proteins using this $5^{\prime}$ sequence (Staub et al., 2000; Tregoning et al., 2003). In the pL3-PpsbA-CT1 vector (Figure 1a), the tobacco promoter and $5^{\prime}$-untranslated region (5'-UTR) of the psbA gene were used. Foreign genes under the control of the psbA promoter and its 5'-UTR are expressed at very high levels (Leelavathi and Reddy, 2003; Molina et al., 2004; Arlen et al., 2007; Fernández-San Millán et al., 2008).

The chimeric genes were cloned into chloroplast transformation vector pL3, developed in this laboratory (Figure $1 \mathrm{~b}$ ). This vector integrates genes of interest into the plastid 
(a) Hybridizing bands

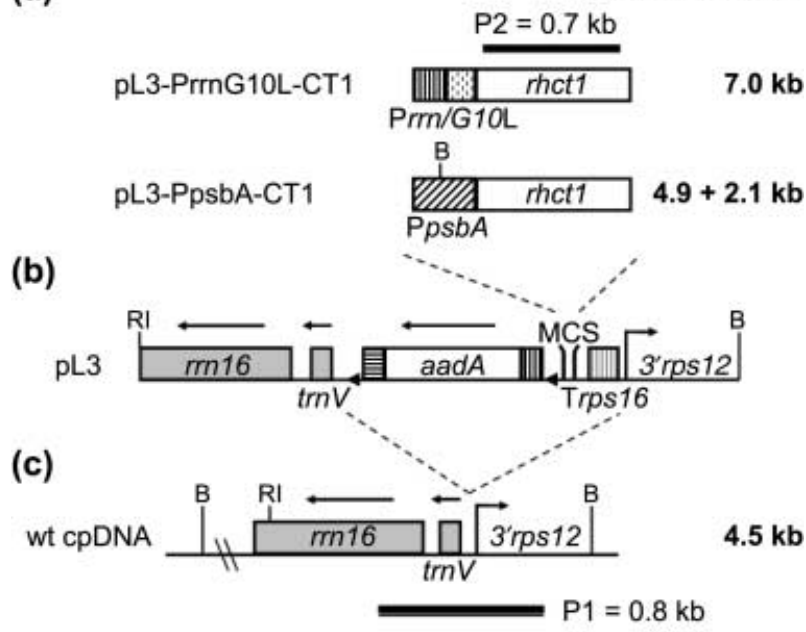

(d)

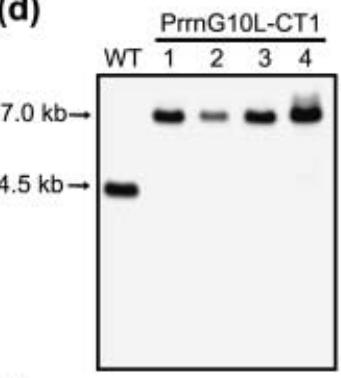

(f)
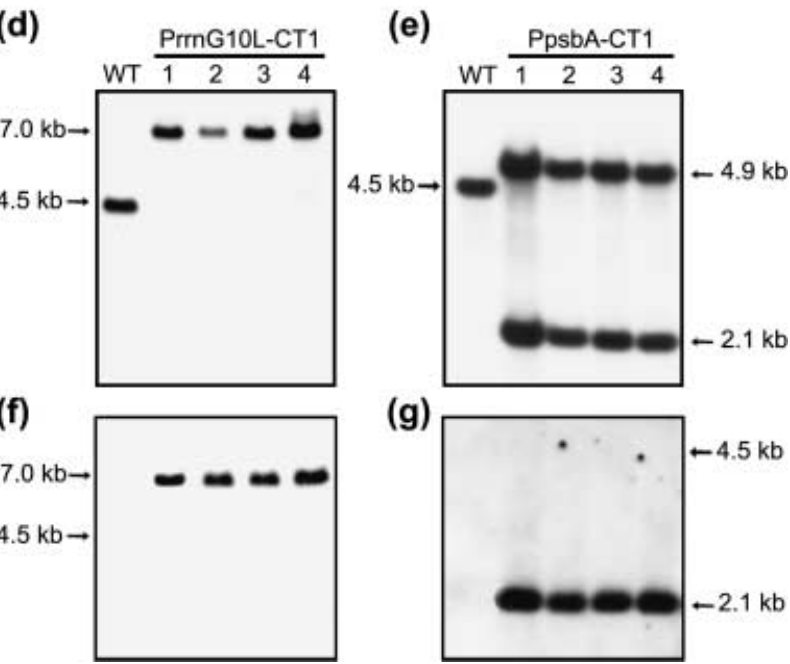

(g)

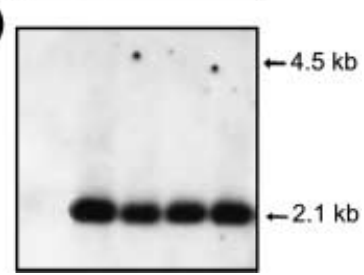

Figure 1 Integration of chimeric rhct1 genes into the chloroplast genome and homoplasmy verification. (a) rhct1 chimeric genes are driven by plastid 5'-expression signals, PpsbA or Prrn/G10L, and the Trps16 trailer needed for mRNA stability. The P2 probe comprised the human cardiotrophin-1 (rhct1) coding region. The numbers to the right indicate the predicted hybridizing bands when digested with $B g / l l$ and probed with P1. (b) Chimeric genes are cloned next to the aadA expression cassette, which is flanked by the loxP sequences (black triangles), in the pL3 plastid transformation vector. The arrows above the boxes show the direction of transcription. (c) Chloroplast genomic sequence at the insertion site between the $\operatorname{trnV}$ and $3^{\prime} r p s 12$ genes. The $0.8-\mathrm{kb}$ fragment (P1) of the targeting region for homologous recombination was used as a probe for Southern blot analysis. $(\mathrm{d}-\mathrm{g})$ Southern blot analysis of four independent lines (1-4) for each chimeric transgene: Prrn/G10L-rhct1$\operatorname{Trps} 16(\mathrm{~d}, \mathrm{f})$ and PpsabA-rhct1-Trps16 (e, g) using the P1 probe $(\mathrm{d}, \mathrm{e})$ or the rhct1 coding region P2 probe (f, g). B, Bglll; MCS, multiple cloning sites; RI, EcoRl; WT, wild-type Petit Havana plant.

genome between the trnV and 3'rps12 genes in the inverted repeat region. These targeting sequences have been widely used in chloroplast transformation and have not exhibited any pleiotropic effect (Maliga, 2004). The chimeric aadA gene, which confers resistance to spectinomycin and streptomycin, was driven by the Prrn promoter and the $p s b A$ terminator. This aadA cassette was flanked by loxP sites, which permit the complete excision of the antibiotic resistance gene after transformation (Lutz and Maliga, 2007). Because the protein synthetic machinery of chloroplasts is similar to that of E. coli, rhCT-1 expression of the pL3-PrrnG10L-CT1 and pL3-PpsbA-CT1 vectors was initially tested in E. coli. Western blot analysis of sonicated $E$. coli cell extracts showed the presence of a 23-kDa rhCT-1, similar to commercially available hCT-1 (data not shown).

The vectors were bombarded into tobacco leaves as described previously (Daniell, 1997). After 5 weeks, several primary shoots appeared from each bombarded leaf as a result of independent transformation events. Putative transformed shoots were identified by growth on $500 \mathrm{mg} / \mathrm{L}$ spectinomycin, and were then submitted to consecutive rounds of regeneration in this selective medium.

\section{Chloroplast integration and homoplasmy verification}

The stable integration of foreign genes into the chloroplast genome and homoplasmy of the transformed plants were confirmed by Southern blot analysis. After three rounds of selection, total leaf DNA from regenerated plants was digested with the Bglll restriction enzyme, which cuts two positions flanking the insertion site outside the left and right recombination regions (Figure $1 \mathrm{c}$ ) and one position in the psbA promoter (Figure 1a). The $0.8-\mathrm{kb}$ probe $\mathrm{P} 1$, which is homologous to the flanking regions rrn $16 /$ trnV and $3^{\prime} r p s 12$ (Figure 1c), was used. Thus, wild-type plastomes release a 4.5-kb DNA fragment, whereas transformed plastomes release either a 7.0-kb DNA fragment (for PrrnG10L-CT1 plants) or two DNA fragments of 4.9 and $2.1 \mathrm{~kb}$ (for PpsbACT1 plants). Four independent lines transformed with each of the transformation vectors were analysed (Figure $1 \mathrm{~d}-\mathrm{g}$ ). The absence of a $4.5-\mathrm{kb}$ fragment in transgenic lines indicates homoplasmy (Figure 1d, e). When the blot was stripped and reprobed with the P2 hCT-1 coding region probe (Figure 1a), hybridization was only detected in the transplastomic lines, as expected (Figure 1f, g). Southern analysis of the $T_{1}$ generation confirmed that all eight transgenic lines analysed maintained homoplasmy (data not shown).

\section{Effect of 5'-regulatory sequences on the expression of} rhCT-1 in tobacco chloroplasts

$\mathrm{T}_{1}$ generation plants were analysed for levels of rhCT-1 accumulation in leaves. Sodium dodecylsulphate-polyacrylamide 

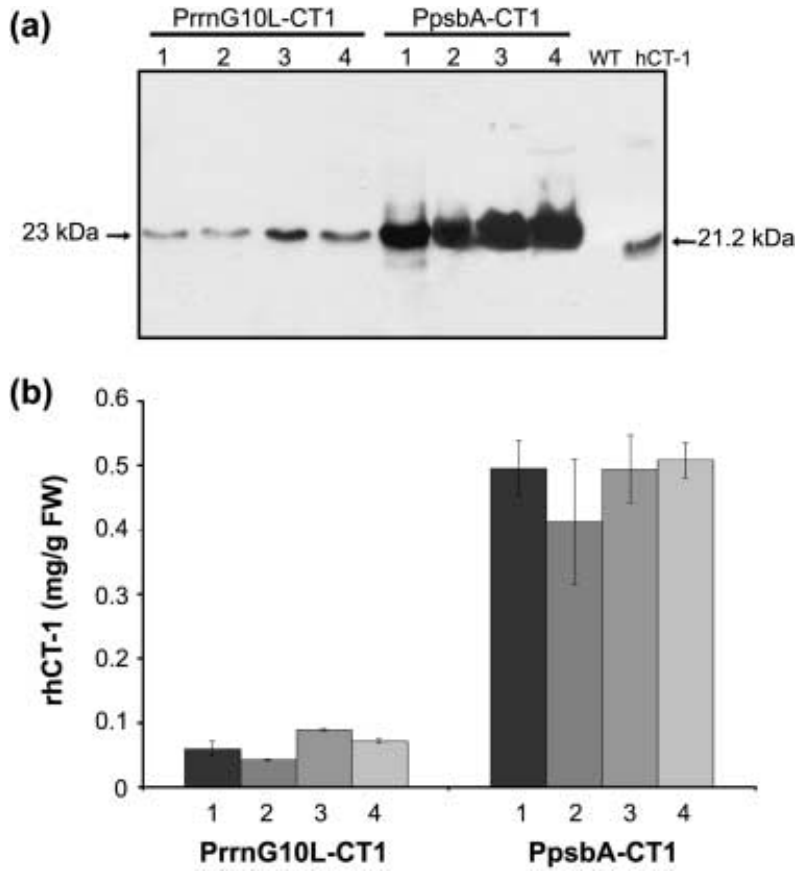

Figure 2 Accumulation of recombinant human cardiotrophin-1 (rhCT-1) protein in tobacco leaves. Four independent lines (1-4) for each construct (PrrnG10L-CT1 and PpsbA-CT1) were analysed. (a) Western blot analysis of rhCT-1. Ten micrograms of total soluble protein were loaded per lane. hCT-1, 75 ng of commercial human cardiotrophin-1 (PeproTech); WT, wild-type Petit Havana plant. (b) Quantification of rhCT-1 expression in transplastomic plants by enzyme-linked immunosorbent assay (ELISA). Total soluble protein was extracted from young, fully expanded leaves of potted plants in the walk-in room harvested 40 days after transplantation. Expressions levels are indicated as milligrams of rhCT-1 per gram leaf fresh weight (FW). The data are presented as the mean \pm standard error of measurements on three individual plants per line.

gel electrophoresis (SDS-PAGE) and Western blot analysis were performed. Monomers of rhCT-1 protein were detected at approximately $23 \mathrm{kDa}$ (Figure 2a), which is slightly larger than the size of commercial hCT-1 $(21.2 \mathrm{kDa})$ as a result of the polyhistidine tag and enterokinase cleavage site in our construct. The signal detected in the Western blot was specific for rhCT-1 because no band was present in the wild-type plant (Figure 2a). Plants transformed with the vector pL3-PpsbA-CT1 accumulated much higher levels of recombinant protein than did plants transformed with pL3-PrrnG10L-CT1 (Figure 2a). This finding is in agreement with results obtained in E. coli, where higher levels of rhCT-1 were found in cell extracts transformed with pL3-PpsbA-CT1 (data not shown).

The levels of recombinant hCT-1 expression in young fully expanded leaves of plants 40 days after transplantation were determined using an enzyme-linked immunosorbent assay (ELISA). Large differences in the accumulation of rhCT-1 were observed between plants transformed with the two different vectors (Figure $2 b$ ), confirming the previous Western blot results. The transplastomic PrrnG10L-CT1 lines accumulated rhCT-1 in leaves at levels ranging from 0.042 to $0.090 \mathrm{mg} / \mathrm{g}$ fresh weight (FW), whereas leaves of PpsbA-CT1 lines produced high levels of recombinant protein, ranging from 0.41 to $0.51 \mathrm{mg} / \mathrm{g}$ FW (Figure 2b). On average, this level of expression is about eight-fold higher than that of the PrrnG10L-CT1 lines. Although very high levels of recombinant protein have been achieved using PrrnG10L (Staub et al., 2000; Tregoning et al., 2003), very low levels (Ye et al., 2001) or levels lower than those obtained with psbA 5'-UTR (Dhingra et al., 2004; Ruhlman et al., 2007) have also been reported. An enhanced translation governed by the G10L $5^{\prime}$-UTR could be gene specific, being critical not only the sequences downstream of the translation initiation codon (Herz et al., 2005), but also each individual mRNA (Kuroda and Maliga, 2001).

Northern blot analysis was performed to determine whether or not changes in rhct1 mRNA accumulation could account for the observed changes in plastid rhCT-1 accumulation. Total cellular RNA extracted from the same leaf samples as used for rhCT-1 quantification was employed for Northern blot analysis. The rhct1 coding region probe showed that transcripts from the psbA promoter were more abundant than those from the rrn promoter in the transplastomic lines (Figure 3, top). Similar findings were obtained by

Figure 3 Steady-state levels of rhct1 mRNA in the transplastomic leaves. Total RNA was extracted from young, fully expanded leaves of potted plants in the walk-in room harvested 40 days after transplantation. Seven plants for each construct (PrrnG10L-CT1 and PpsbA-CT1) were analysed by Northern blot. An aliquot of $5 \mu \mathrm{g}$ of total cellular RNA was loaded per lane. Blots were probed for human cardiotrophin-1 (hCT-1) (top) and 16S rRNA (bottom, loading control). WT, wild-type Petit Havana plant.
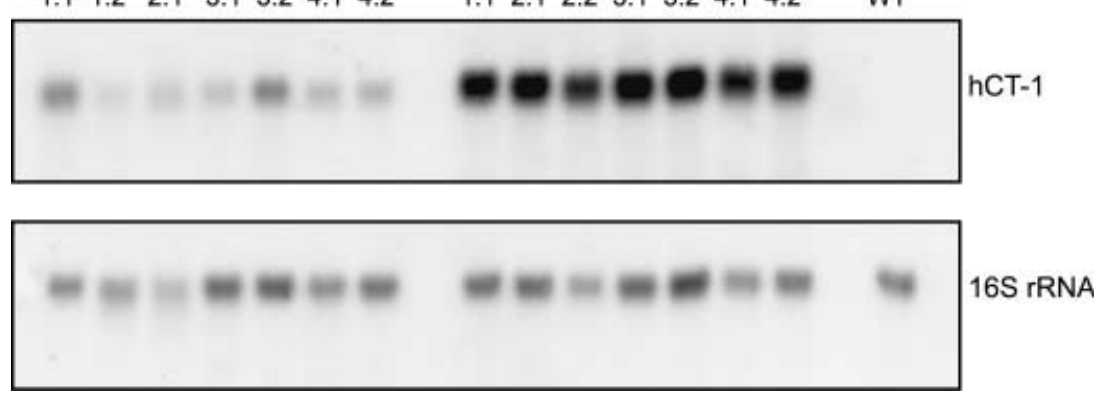


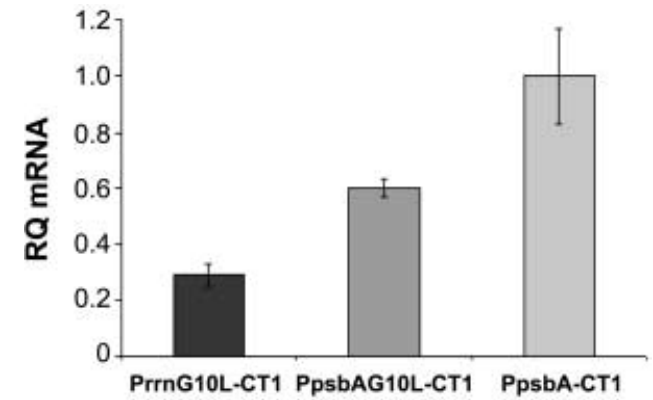

Figure 4 Relative amount of rhct1 mRNA in leaves of transplastomic plants measured by quantitative real-time polymerase chain reaction. Data are normalized to the plastid aadA transcript and computed relative to the PpsbA-CT1 value, which was set to unity. Data are presented as the mean \pm standard error of measurements from 9-12 samples (three to four independent plants per three biological replicates) per construct.

Dhingra et al. (2004). To rule out any potential loading artefact, the Northern blot was stripped and reprobed with a 165 rRNA-encoding DNA fragment (Figure 3, bottom). In this study, the chimeric genes have the same coding region (rhct1) and 3'-UTR (Trps16), but differ in the promoter (PpsbA and Prrn) and 5'-UTR (psbA and G10L). Therefore, the increase in mRNA levels may be caused by either an increase in transcription rate or mRNA stability because of the leader sequence.

To study the significance of a putative interaction between promoter and 5'-UTR and how this could affect rhCT-1 accumulation in chloroplasts, a new transplastomic plant was constructed expressing rhct 1 under the control of a chimeric promoter that combines the psbA promoter with the $\mathrm{G} 10 \mathrm{~L}$ $5^{\prime}$-UTR. This chimeric gene was cloned into the chloroplast transformation vector pL3, and bombarded into tobacco leaves as described above, to obtain homoplasmic PpsbAG10LCT1 plants (Figure S1, see 'Supplementary material'). Real-time polymerase chain reaction (PCR) was performed to quantify the rhct1 mRNA accumulated in these plants, and was compared with that produced by PpsbA-CT1 and PrrnG10LCT1 plants. Real-time PCR results indicated that rhct1 transcripts of PpsbA-CT1 plants were 1.7-fold more abundant than those of PpsbAG10L-CT1 plants (Figure 4). Although these plants expressed rhCT-1 under the control of the same promoter ( $p s b A)$, the mRNA increase could be explained by a greater stability of the transgene transcript conferred by the psbA 5'-UTR. Several authors have already reported a high stability of psbA transcripts in mature leaves (Kim et al., 1993; Zoschke et al., 2007), and the importance of the psbA 5'-UTR for mRNA stabilization and translation efficiency (Zou et al., 2003). Likewise, rhct1 transcripts from PpsbAG10L were two-fold more abundant than those from PrrnG10L (Figure 4). In this case, both transcripts share the
G10L 5'-UTR, and therefore their mRNA stability should be identical. Thus, the observed mRNA differences could only be attributed to the promoter strength. Several authors have reported that $16 \mathrm{~S}$ rRNA has a higher transcription rate than psbA early in chloroplast development, but lower in mature chloroplasts (Baumgartner et al., 1993; DuBell and Mullet, 1995). This change is consistent with the protein synthesis mechanisms of chloroplasts. Thus, although the synthesis of the plastid decoding apparatus occurs early in chloroplast development, the ability of this machinery to synthesize photosynthetic proteins takes place in the mature chloroplast. Recently, Zoschke et al. (2007) have also shown that psbA transcription rates in young and mature leaves of Arabidopsis plants are two- to three-fold higher than those of the $r r n 16$ gene. Therefore, it is concluded that, under our conditions, the $p s b A$ promoter is stronger than the $r r n$ promoter, and the psbA 5'-UTR confers more stability than the G10L 5'-UTR to the transgene transcript.

The protein accumulated in PpsbAG10L-CT1 plants (0.07 $\pm 0.01 \mathrm{mg} / \mathrm{g}$ FW) matched that of PrrnG10L-CT1 plants (Figure 2b), and both were much lower than that in PpsbACT1 plants (Figure 2b). Thus, protein levels seemed to be influenced more by the $5^{\prime}$-UTR than by mRNA abundance. Such a lack of correlation between transcript abundance and protein accumulation has been reported previously when the psbA 5'-UTR was used (Staub and Maliga, 1994; FernándezSan Millán et al., 2003), suggesting an important role of the psbA 5'-UTR in the enhancement of translation (Eibl et al., 1999). Recently, Yukawa et al. (2007) have shown that the 5 '-UTR of the psbA gene results in the most efficient translation rate of 11 tobacco plastid genes studied. In this work, it was clearly demonstrated that the relative rhct1 translation efficiency of the psbA $5^{\prime}$-UTR was much higher than that of the G10L 5'-UTR. In addition, it was shown that the translatability of the mRNA, determined by the $5^{\prime}$-translation control elements, was more important for chloroplast protein expression than mRNA abundance. Indeed, the choice of $5^{\prime}$-UTR may affect protein yields over a 10 000-fold range (Maliga, 2003). It is thus advisable, in order to increase protein yields, to test different $5^{\prime}$-UTR sequences before proceeding with the expression of a new transgene in the chloroplast.

\section{Characterization of rhCT-1 in transgenic plants}

The external appearance, age and environmental factors, such as lighting and temperature, contribute to the levels of expressed protein (Stevens et al., 2000; Arlen et al., 2007). As mentioned above, two different $5^{\prime}$-UTRs were used in this study to enhance the translation of rhct1 CDNA. The T7 G10L 
5'-UTR appears to facilitate constitutive translation of the transgene (Kumar et al., 2004), whereas the translation of psbA 5'-UTR is regulated by light in tobacco plastids (Staub and Maliga, 1994). First, the changes in rhCT-1 levels were examined under different light regimes, comparing the expression levels in transplastomic plants (previously grown under a 16-h light/8-h dark cycle) when placed for $20 \mathrm{~h}$ in darkness or 12, 24, 32 and $50 \mathrm{~h}$ in continuous light. No significant changes in rhCT-1 level were observed between darkness and $12 \mathrm{~h}$ of light (Figure $5 \mathrm{a}$ ), suggesting that the decrease in translation efficiency by psbA $5^{\prime}$-UTR in darkness

(a)

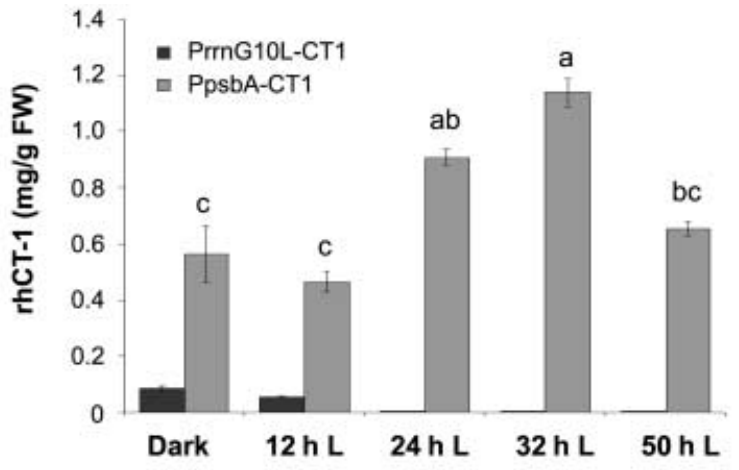

(b)

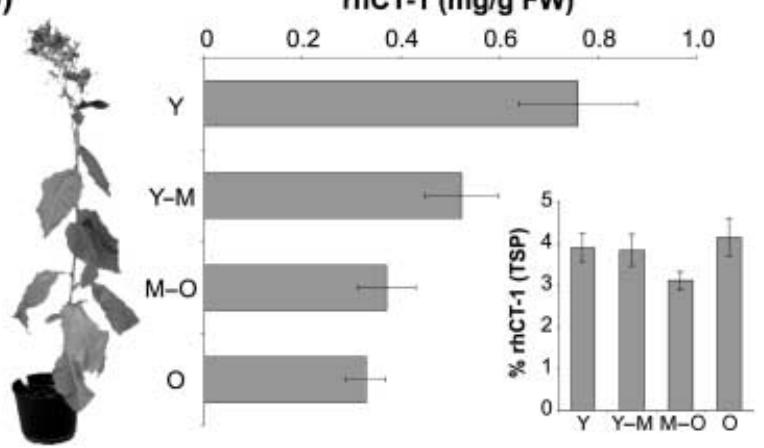

Figure 5 Characterization of transgenic recombinant human cardiotrophin-1 (rhCT-1)-producing plants. (a) Study after different hours of lighting. Samples of leaves were collected from potted plants transformed with PrrnG10L-CT1 or PpsbA-CT1 after a 20-h dark period or at the indicated hours of light (L). Expressions levels are indicated as milligrams of rhCT-1 per gram fresh weight (FW). Data are presented as the means \pm standard error of measurements on four individual plants per construct. Different letters indicate statistically different values at $P=0.05$ using the Duncan test. (b) rhCT-1 accumulation in leaves of potted plants in the walk-in room at different developmental stages. The plant to the left indicates the respective positions of the different leaves $(\mathrm{Y}, \mathrm{Y}-\mathrm{M}, \mathrm{M}-\mathrm{O}, \mathrm{O})$ in the plant. Total soluble protein was extracted from PpsbA-CT1 transformed plants and quantified by enzyme-linked immunosorbent assay (ELISA). Expressions levels are indicated as milligrams of rhCT-1 per gram FW, and as percentage of total soluble protein (TSP) (bottom right-hand corner). The data are presented as the mean \pm standard error of measurements on four individual plants.

$\mathrm{Y}$, young leaves; $\mathrm{M}$, mature leaves; $\mathrm{O}$, old leaves.
(Trebitsh et al., 2000) may be counterbalanced by an increase in protein stability under these conditions (Wirth et al., 2006). Under continuous light, the levels of rhCT-1 accumulated in the leaves of PrrnG10L-CT1 plants were below the detection limit of ELISA ( $2 \mu \mathrm{g} / \mathrm{g}$ FW). Nevertheless, rhCT-1 produced in leaves of PpsbA-CT1 plants accumulated as the continuous light exposure increased, reaching a peak after $32 \mathrm{~h}$ of continuous illumination (Figure $5 \mathrm{a}$ ), with a yield of $1.14 \mathrm{mg} / \mathrm{g}$ FW, equivalent to $4.8 \%$ of TSP. Several studies have demonstrated that the psbA 5'-UTR confers light-dependent accumulation to other foreign proteins (Fernández-San Millán et al., 2003; Dhingra et al., 2004; Watson et al., 2004; Arlen et al., 2007). Notwithstanding this, the levels of rhCT-1 decreased after $50 \mathrm{~h}$ of continuous light exposure (Figure $5 \mathrm{a}$ ). Similar observations have been reported by other authors (Dhingra et al., 2004; Arlen et al., 2007). The rhCT-1 decrease detected after $50 \mathrm{~h}$ of continuous light could reflect either a reduction in protein synthesis or a higher protein turnover induced by over-lighting conditions (Zheng et al., 2002).

To examine the variation in rhCT-1 levels in differently developed leaves, TSP extracts from the highest expressing transgenic PpsbA-CT1 plant were prepared from leaves harvested at different positions along the plant, and subjected to ELISA. The relative amount of rhCT-1 protein per gram FW was highest in the youngest leaves (Figure 5b). Old senescent leaves showed much smaller amounts of recombinant protein, about $50 \%$ of that in young leaves (Figure $5 \mathrm{~b}$ ). The content of TSP also decreased from $23 \mathrm{mg} / \mathrm{g}$ FW in young leaves to $9 \mathrm{mg} / \mathrm{g}$ FW in old leaves, and the profiles of recombinant protein matched those of TSP. The amount of rhCT-1 expressed as a percentage of TSP remained basically unchanged (Figure 5b), as described previously (Stevens et al., 2000; Molina et al., 2004). The lower content of recombinant protein in older leaves of transgenic plants is not surprising because senescence activates proteolytic activity (Sakamoto, 2006), which degrades both endogenous and foreign proteins. However, several studies have reported that optimal levels of expression can be achieved in mature and old leaves of fully grown plants (Staub et al., 2000; De Cosa et al., 2001; Fernández-San Millán et al., 2003). In these reports, the recombinant protein formed inclusion bodies (Fernández-San Millán et al., 2003), folded into stable crystalline structures (De Cosa et al., 2001) or aggregated via a chaperonin-like effect of ubiquitin (Staub et al., 2000), protecting them from degradation. When a protein more susceptible to degradation is expressed in plastids, the highest yields are obtained in fast-growing, young leaves, whereas no protein or very low levels are present in mature leaves (Birch-Machin et al., 2004; Herz et al., 2005). 
The expression level of recombinant protein is thus highly dependent on tissue type, plant age and culture conditions. For the assessment of cost-effective protein production in plant plastids, all of these factors should be taken into account. In our work, higher levels of recombinant accumulation were achieved in fast-growing, young leaves of fully grown plants harvested after $32 \mathrm{~h}$ of continuous light.

\section{Plastid-expressed hCT-1 is bioactive}

To test the biological activity of recombinant hCT-1 produced in chloroplasts, a human hepatocarcinoma cell line, HepG2, was used. In this cell line, the ability of rhCT-1 to induce phosphorylation of the signal transducer and activator of transcription-3 (STAT-3) was measured. Non-stimulated cells were used as a negative control. Phosphorylation of STAT-3 (P-STAT-3) was assessed by immunoblot analysis. The crude extracts from wild-type and transplastomic PpsbA-CT1 plants were added to the HepG2 cell culture medium. As a positive control, commercial hCT-1 was used. Wild-type plant extract showed no activity in this assay (Figure 6a, top), indicating that there is no endogenous plant compound that can phosphorylate the STAT-3 protein. In contrast, the PpsbACT1 plant extract induced such phosphorylation, although the extent of P-STAT-3 induction by transgenic plant extracts, harvested after $30 \mathrm{~h}$ of continuous light, was significantly lower than that expected from the recombinant protein concentration used in the assay (Figure 6a, top). In addition, the pattern shown by actin band intensities (Figure 6a, bottom) indicates that the observed differences in P-STAT-3 levels are not a result of loading variability. Densitometric analysis was performed to quantify the P-STAT-3 protein. Although rhCT-1 amounts increased by over two-fold under $32 \mathrm{~h}$ of continuous light (Figure $5 \mathrm{a}$ ), the ability of rhCT-1 obtained from these plants to induce P-STAT-3 was significantly lower $(\sim 35 \%)$ than the ability of commercial hCT-1 (Figure 6b). However, only a slight decrease in rhCT-1 bioactivity was observed when leaves were harvested under dark conditions, or after $12 \mathrm{~h}$ of light (Figure $6 \mathrm{~b}$ ). One of the most deleterious effects of environmental stress (i.e. continuous light) on plants is 'photo-oxidative stress', which is primarily induced by the large absorption of excitation energy, leading to over-reduction of the electron transport chains. As a result, harmful reactive oxygen species (ROS) build up in tissues (Reddy and Raghavendra, 2006), therefore probably affecting rhCT-1 bioactivity. These oxidative conditions could also alter rhCT-1 folding and bioactivity. In this context, it is interesting to note that no experimental evidence exists on disulphide bridge formation in human CT-1 protein.
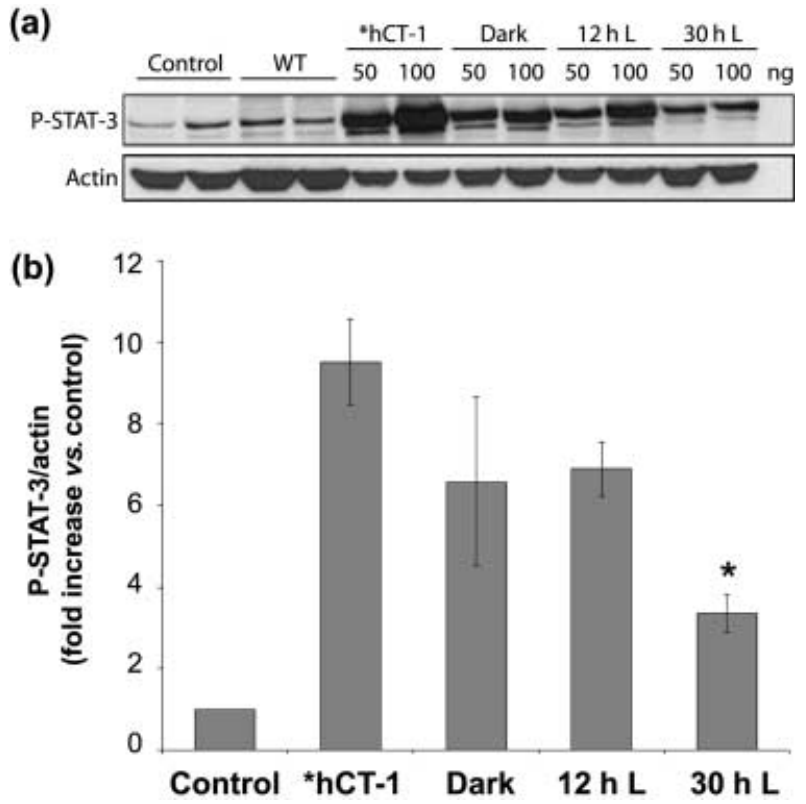

Figure 6 Bioactivity of recombinant human cardiotrophin-1 (rhCT-1) produced in tobacco chloroplasts. (a) Representative Western blot for signal transducer and activator of transcription-3 phosphorylation ( $\mathrm{P}$ STAT-3) (Tyr705) and actin (loading control) in HepG2 cell line stimulated with 50 and 100 ng per well of commercial hCT-1 (*hCT-1) or equivalent recombinant hCT-1 amounts from the crude extract of PpsbA-CT1 leaves harvested in darkness (Dark) of under $12 \mathrm{~h} \mathrm{(12} \mathrm{h} \mathrm{L)} \mathrm{and} 30 \mathrm{~h} \mathrm{(30} \mathrm{h} \mathrm{L)} \mathrm{of}$ light. Cells treated with wild-type crude extract (WT) and non-stimulated cells (Control) were used as negative controls. (b) Densitometric analysis of P-STAT-3 in stimulated HepG2 cell line extracts. The expression levels are presented as the fold increase vs. control $(=1)$ using the ratios between the densities of the P-STAT-3 bands and the corresponding actin bands. The data are presented as the mean \pm standard error of two individual experiments. ${ }^{*} P=0.1$ vs. commercial hCT-1.

In addition, published CT-1 sequences, such as those from rat and mouse, have only one cysteine, suggesting that the disulphide bridge linkage is not essential for activity. Perhaps the over-oxidizing conditions arising under $32 \mathrm{~h}$ of continuous light could lead to modifications of the recombinant protein structure and its bioactivity. Such a decrease in protein bioactivity has never been described previously in chloroplasts expressing therapeutic proteins under continuous light (Arlen et al., 2007; Ruhlman et al., 2007). To our knowledge, this is the first time that the bioactivity of a chloroplast-made therapeutic protein has been shown to be significantly affected by over-lighting, probably as a result of specific features of the CT-1 protein. Indeed, oxidative stress has been found to stimulate CT-1 release to the extracellular milieu from isolated hepatocytes (Iñiguez et al., 2006), whereas, under non-stress conditions, CT-1 is found inside the cell. Ateghang et al. (2006) also described the regulation of CT-1 expression in mouse embryonic stem cells by 
(a)

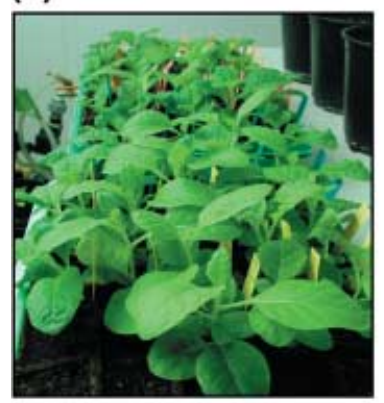

(b)

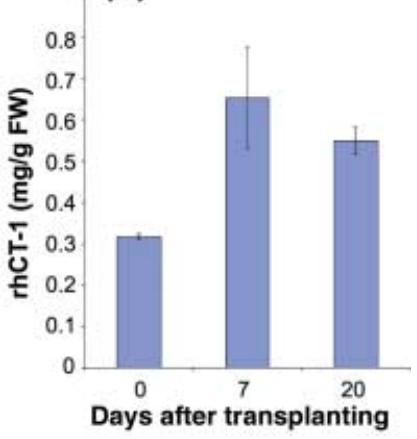

Figure 7 Production of recombinant human cardiotrophin-1 (rhCT-1) in seedlings. (a) Seedlings, germinated and grown in vitro for 4 weeks, were transferred to soil in jiffy pots until harvest. (b) Quantification of rhCT-1 expression by enzyme-linked immunosorbent assay (ELISA). Total soluble protein was extracted from seedling plants grown in a walk-in room and harvested at different days after transplantation (0, 7 and 20 days). Expression levels are indicated as milligrams of rhCT-1 per gram fresh weight (FW). The data are presented as the mean \pm standard error of measurements on four individual seedling plants.

intracellular ROS. To assess these results, further studies will be specifically addressed in future research.

\section{Scaling up the production of rhCT-1 in seedlings}

To evaluate the feasibility of using these plants for commercial purposes, the production of rhCT-1 in seedlings grown in a walk-in room under a 16 -h photoperiod was also examined (Figure 7a). The amount of rhCT-1 accumulated in PpsbA-CT1 plantlets harvested at different times after transplantation was quantified by ELISA (Figure 7b). Levels of rhCT-1 produced in 7- and 20-day-old plantlets (0.65 and $0.55 \mathrm{mg} / \mathrm{g} \mathrm{FW}$, respectively) were approximately two-fold higher than those produced in plantlets just after in vitro culture $(0.32 \mathrm{mg} / \mathrm{g} \mathrm{FW})$.

A 'closed commercial production system' was commercialized in Japan in 2002 for the production of horticultural crop seedlings (Kozai, 2005). This system offers several advantages over seedling production in glasshouses. The most important is the higher productivity $(\sim 10$-fold) per floor area per year, an effect that is mainly attributable to the high plant density (up to 1500 seedlings $/ \mathrm{m}^{2}$ ) and shorter production period (20 days). Each unit of the commercial-type closed system has a floor area of $22 \mathrm{~m}^{2}$, thus producing 33000 seedlings in one batch; this amounts to 594000 seedlings per year (one cycle lasts 20 days, permitting 18 batches/year) (Kozai, 2005). We were able to generate 4.2 mg of rhCT-1 from a single plantlet grown in a walk-in room when harvested 20 days after transplantation (Table 1). From the observed expression levels, it should be possible to obtain yields of up to $2.5 \mathrm{~kg}$ of rhCT-1 per unit of the commercial-type closed system and year, taking into account the high plant productivity described above. Furthermore, by increasing the harvest frequency to 7 days, despite decreasing yields per plantlet (1.9 mg/plantlet; Table 1), it should be possible to produce up to $3.2 \mathrm{~kg}$ of rhCT-1 per unit of the commercial-type closed system and year (52 batches/year). One of the potential therapeutic uses of CT-1 is as a hepatoprotective in liver transplantation surgery (Bustos et al., 2003) (procedures that are carried out at a rate of about 1000 per year in Spain, 4500 in the USA, 5000 in Europe and more than 15000 per year all around the world). A dose of recombinant hCT-1 for this purpose could be about 14-28 mg for an average 70-kg individual. Based on a potential purification efficiency of $50 \%$, more than 45000 doses per year could be produced in a single commercial-type closed system, a yield that far exceeds the estimated worldwide needs of hCT-1 for liver transplantation surgery. However, this cytokine might also be applied as a tissue protective factor against ischaemiareperfusion injury in the transplantation of other organs (heart, kidney, etc.), and to preserve the function of the remnant liver after surgery for primary and metastatic liver cancer (Bustos et al., 2003). New potential therapeutic uses continue to appear (e.g. Alzheimer's disease), raising interest in the development of efficient production systems.

The commercial potential of molecular farming of plantmade pharmaceuticals is regulated to ensure the protection of both the environment and consumers. The chloroplast transformation system provides for transgene containment from pollen transmission. However, two recent articles (Ruf et al., 2007; Svab and Maliga, 2007) have reported pollen transmission in the range $0.0087 \%-0.00024 \%$ in tobacco
Table 1 Yield of recombinant human cardiotrophin-1 (rhCT-1) protein expressed in chloroplasts of transgenic tobacco plantlets grown in a walk-in room

\begin{tabular}{llll}
\hline $\begin{array}{l}\text { Plantlet age (days after } \\
\text { transplantation) }\end{array}$ & $\begin{array}{l}\text { rhCT-1 in fresh } \\
\text { plantlet }(\mathrm{mg} / \mathrm{g})\end{array}$ & $\begin{array}{l}\text { Average weight } \\
\text { per plantlet }(\mathrm{g})\end{array}$ & $\begin{array}{l}\text { Yield of rhCT-1 } \\
\text { (mg/plantlet) }\end{array}$ \\
\hline 7 & $0.65 \pm 0.12(n=4)$ & $2.9 \pm 0.36(n=10)$ & 1.9 \\
20 & $0.55 \pm 0.03(n=4)$ & $7.7 \pm 0.64(n=10)$ & 4.2 \\
\hline
\end{tabular}

Data are the mean \pm standard error; number of replications given in parentheses. 
plants. In our study, a confined method is proposed for the production of recombinant proteins in seedling plants in an environmentally friendly manner, thereby eliminating the potential hazard of pollen or seed spread.

In conclusion, a high-density seedling expression system is described to over-produce bioactive rhCT-1, as a His-tag fusion protein with enterokinase cleavage site, in tobacco chloroplasts. Although no attempt has yet been made to cleave the purification tag, the expression of this fusion protein will probably allow the production of tag-free pure fractions, which is essential for further clinical studies. The plastid transformation vector used in this work is equipped with loxP sequences, thus providing an efficient system to obtain transplastomic plants free of the aadA spectinomycin resistance gene.

\section{Experimental procedures}

\section{Genetic engineering of chloroplast expression vectors}

Tobacco chloroplast transformation vectors were based on pBluescript SK (Stratagene, La Jolla, CA, USA), with the elements necessary for plastid transformation inserted between the Sacl and Kpnl restriction sites. The aadA coding sequence fused to the rrn promoter (PrrnaadA) was obtained from plasmid pFaadAll (Eibl et al., 1999). rhct1 (bp 38-643 according to GENBANK accession number BC064416) was amplified by PCR from the IRAKp961M02139Q clone (RZPD Deutsches Ressourcenzentrum für Genomforschung $\mathrm{GmbH}$, Berlin, Germany). Other elements were amplified by PCR from Nicotiana tabacum plastid DNA (bp positions according to GENBANK accession number NC_001879): flanking regions, bp 138 544-140 315 and 140 316-141 475; psbA termination sequence (TpsbA), bp 140-534; rps16 termination sequence (Trps16), bp 4939-5087; psbA promoter and its 5'-UTR (PpsbA), bp 1598-1785; 16 S rRNA promoter (Prrn), bp 139 982-140 065; psbA promoter, bp 1683-1785. The complete aadA selection cassette was flanked by two loxP sites inserted by cloning primers. The His tag enterokinase sequence (5'-ATGGGTCACCATCACCATCACCATGACGATGACGATAAG-3') and the leader sequence of phage T7 G10L (bp 22 904-22 966 according to GENBANK accession number NC_001604) were amplified by PCR-based gene assembly using overlapping primers. Finally, PrrnG10L and PpsbAG10L were produced by overlap extension PCR. The complete sequences of the final vectors pL3-PpsbA-CT1, pL3-PrrnG10L-CT1 and pL3-PpsbAG10L-CT1 are available from GENBANK (accession numbers EU520589, EU520587 and EU520588, respectively). Construction details are available on request. Before proceeding with bombardment, the vectors were tested by Western blot analysis in E. coli.

\section{Bombardment and regeneration of chloroplast transgenic plants}

Gold microprojectiles coated with plasmid DNA (pL3-PpsbA-CT1, pL3-PrrnG10L-CT1 or pL3-PpsbAG10L-CT1) were bombarded into tobacco (N. tabacum var. Petit Havana) in vitro-grown leaves using the PDS1000/He (Bio-Rad, Hercules, CA, USA) biolistic device, as described previously (Daniell, 1997). After bombardment, the leaves were incubated in the dark for 2 days at $28^{\circ} \mathrm{C}$. The leaves were then cut into small pieces $(\sim 5 \mathrm{~mm} \times 5 \mathrm{~mm})$ and placed, adaxial side up, on selection medium (RMOP) in Magenta vessels (Sigma, St Louis, MO, USA) containing 500 mg/L spectinomycin dihydrochloride as selecting agent. The growth conditions of the culture chamber were $28{ }^{\circ} \mathrm{C}, 120 \mu \mathrm{mol} / \mathrm{m}^{2} / \mathrm{s}$ and a 16 -h photoperiod. Spectinomycinresistant shoots obtained after $6-7$ weeks were cut into small pieces $(\sim 2 \mathrm{~mm} \times 2 \mathrm{~mm})$ and subjected to successive rounds of selection in the same selection medium. After three to four rounds, regenerated plants were transplanted and grown in a walk-in room under the following conditions: $28{ }^{\circ} \mathrm{C}, 250 \mu \mathrm{mol} / \mathrm{m}^{2} / \mathrm{s}, 70 \%$ relative humidity and $16-\mathrm{h}$ photoperiod.

\section{Southern and Northern blot analyses}

Total plant DNA $(10 \mu \mathrm{g})$ was digested with Bg/ll, separated on a $0.8 \%(\mathrm{w} / \mathrm{V})$ agarose gel and transferred to a nylon membrane. A $0.8-k b$ probe (P1), homologous to the flanking sequences, was obtained by PCR using tobacco DNA as template and the following primers: 5'-CCAAGGCATAACTTGTATCC-3' and 5'-GAGTGTACCATGAGAGAAGC-3'. The PSK-PrrnG10L-CT1 vector, digested with Sall and Ncol, generated a $0.7-k b$ probe (P2) homologous to the rhct1 sequence. Probe labelling and hybridization were performed using the chemiluminescent AlkPhos direct labelling detection system (GE Healthcare, Buckinghamshire, UK). After Southern blot analysis confirmation, the plants were transferred to soil. Seeds from the $T_{0}$ generation were in vitro germinated on spectinomycin selection medium. The $T_{1}$ seedlings were isolated and cultured for 2 weeks in Magenta vessels. Finally, plants were transferred to pots. Plants of the $T_{0}$ and $T_{1}$ generations were analysed for homoplasmy.

Total RNA was extracted (Ultraspec RNA, Biotecx Laboratories, Houston, TX, USA) from the leaves of transformed and untransformed plants. RNA $(5 \mu \mathrm{g})$ was separated on $1.5 \%$ agarose/formaldehyde gels and then transferred to a nylon membrane. P2 was used as the hCT-1-specific probe (described above). Constitutive $16 \mathrm{~S}$ probe (150-bp) was generated by PCR using tobacco DNA as template and the following primers: 5'-CTTTTTAAGTCCGCCGTCAAA-3' and 5'-TCTTTCCGATCTCTAC GCATTTC-3'. Labelling and hybridization were performed using the chemiluminescent detection system mentioned above.

\section{Transcript quantification by real-time PCR}

Transcript quantification was performed by real-time PCR. Four independent plants for the PpsbA-CT1 and PrrnG10L-CT1 constructs (three independent plants for PpsbAG10L-CT1) and three biological replicates per plant were analysed. Untransformed plants grown under the same conditions were used as controls. Total RNA was extracted (Ultraspec RNA, Biotecx Laboratories) and analysed by the SyBR Green protocol (Applied Biosystems, Foster City, CA, USA) at Scientific Park (Universidad Autonoma de Madrid, Madrid, Spain). The primers for rhct 1 detection were $5^{\prime}$-TCTCACTTCTTCCCCACTTGGA-3' and $5^{\prime}$-CAAGGCTGTGTGTCTGACGG-3'. The aadA transcript was used as constitutive control of the plastid RNA loaded per reaction. The primers for aadA detection were 5'-ATCATTCCGTGGCGTTATCC-3' and $5^{\prime}$-CCAAGGCAACGCTATGTTCTC-3'. 


\section{Western blot analysis}

Transformed and untransformed leaves of plants grown in a walk-in room were ground in liquid nitrogen. TSP was extracted as described previously (Farran et al., 2002). Leaf extracts were electrophoresed in a $13 \%$ polyacrylamide gel, and separated proteins were transferred to a nitrocellulose membrane for immunoblotting. Commercial hCT-1 protein (PeproTech, London, UK) was used as positive control. The primary antibody (monoclonal anti-hCT-1; R\&D Systems, Minneapolis, MN, USA) was used at a $1: 2000$ dilution, and the secondary antibody (horseradish peroxidase-conjugated goat anti-mouse immunoglobulin G; Pierce, Rockford, IL, USA) was used at a $1: 5000$ dilution. Detection was performed using the enhanced chemiluminescence (ECL) Western blotting system (GE Healthcare).

\section{ELISA quantification of recombinant protein}

Transformed and untransformed leaves (100 mg of each) from plants grown in a walk-in room were ground in liquid nitrogen, resuspended in $500 \mu \mathrm{L}$ of extraction buffer $(20 \mathrm{~mm}$ sodium phosphate, $\mathrm{pH} 7.4,500 \mathrm{~mm} \mathrm{NaCl}, 0.1 \%$ Triton $\mathrm{X}-100$ and cocktail of anti-protease) and incubated for $30 \mathrm{~min}$ on ice. The supernatant obtained after $10 \mathrm{~min}$ of centrifugation at $20800 \mathrm{~g}$ contained the soluble fraction. The process was performed at $4{ }^{\circ} \mathrm{C}$. Sandwich ELISA was used to determine the recombinant hCT-1 concentration in these samples. Capture antibody (monoclonal anti-hCT-1 antibody; R\&D Systems), at $4 \mu \mathrm{g} / \mathrm{mL}$ of phosphate-buffered saline (PBS), was bound to a 96-well polyvinyl chloride microtitre plate (Costar Corning, Corning, NY, USA) overnight at room temperature. Background was blocked with $1 \%(\mathrm{w} / \mathrm{v})$ bovine serum albumin (BSA) and sucrose in PBS for $2 \mathrm{~h}$ at room temperature. Plates were incubated with samples or standards diluted in buffer [ $20 \mathrm{mM}$ tris(hydroxymethyl)aminomethane (Tris)- $\mathrm{HCl}, \mathrm{pH} 7.4,150 \mathrm{~mm} \mathrm{NaCl}, 0.1 \%$ BSA, 0.05\% Tween-20] for $2 \mathrm{~h}$ at room temperature. The plates were then incubated with $200 \mathrm{ng} / \mathrm{mL}$ of a biotinylated anti-hCT-1 antibody (R\&D Systems) in blocking solution ( $2 \mathrm{~h}$ at room temperature), followed by incubation with streptavidin-horseradish peroxidase conjugate (Amersham) for $1 \mathrm{~h}$ at room temperature. Washes were performed between each step with PBS containing Tween-20 (0.1\%). Colour was developed by adding 2,2'-azino-bis(3-ethylbenzthiazoline-6sulphonic) acid (ABTS; Roche, Mannheim, Germany), and the reactions were terminated after $1 \mathrm{~h}$ with $1 \%$ SDS. The absorbance at $405 \mathrm{~nm}$ was measured in a microplate reader (Multiskan Ex, Labsystems, Helsinki, Finland). A standard curve to calculate the amount of recombinant protein was made by plating a commercial hCT-1 (PeproTech) over a range of 40-125 ng per well. Transgenic leaf extracts were diluted to fit into the linear range of the hCT-1 standard. The total protein content of extracts was measured by the Bradford assay (Bio-Rad).

\section{Bioactivity analysis of recombinant hCT-1}

The human hepatoma cell line HepG2 was maintained in Dulbecco's modified Eagle's medium (Gibco, Paisley, UK) containing 10\% fetal calf serum. For cell signalling experiments, cells were coated in sixwell plates with $7.5 \times 10^{5}$ cells/well. After adhesion, cells were cultured in serum-free medium for $48 \mathrm{~h}$, and then stimulated for 30 min with different amounts of commercial hCT-1 (PeproTech) or rhCT-1 from crude extracts of PpsbA-CT1s plants. Cells treated with wild-type plant extract and non-stimulated cells were used as negative controls. HepG2 cells were lysed for Western blot analysis in cold lysis buffer ( $50 \mathrm{~mm}$ Tris- $\mathrm{HCl}, \mathrm{pH} 7.4,150 \mathrm{~mm} \mathrm{KCl}, 1 \%$ Nonidet P-40, 0.5\% sodium deoxycholate, $0.1 \%$ SDS, $1 \mathrm{~mm}$ sodium orthovanadate, $10 \mathrm{~mm}$ sodium fluoride and cocktail of anti-proteases). Protein from lysates was heat denatured in double-strength SDS sample buffer containing dithiothreitol before resolution by SDSPAGE. Western blot analysis was performed using specific antibodies for phosphorylated STAT-3 (Tyr705) (Cell Signalling, Beverly, MA, USA) and actin (Sigma). Densitometric analysis was performed by chemiluminescence detection using an ImageQuant ECL system and ImageQuant TL software (GE Healthcare). The expression levels are presented as the fold increase vs. control $(=1)$ using the ratios between the densities of the P-STAT-3 bands and the corresponding actin bands.

\section{Acknowledgements}

The authors are grateful to Christian Eibl for providing pFaadAll. They thank Jon Veramendi and Patricia Obregon for critical reading of the manuscript. This work was supported by a Grant from the Departamento de Educación y Cultura (Gobierno de Navarra).

\section{References}

Arlen, P.A., Falconer, R., Cherukumilli, S., Cole, A., Cole, A.M., Oishi, K.K. and Daniell, H. (2007) Field production and functional evaluation of chloroplast-derived interferon-alpha2b. Plant Biotechnol. J. 5, 511-525.

Ateghang, B., Wartenberg, M., Gassmann, M. and Sauer, H. (2006) Regulation of cardiotrophin-1 expression in mouse embryonic stem cells by HIF-1alpha and intracellular reactive oxygen species. J. Cell Sci. 119, 1043-1052.

Baumgartner, B.J., Rapp, J.C. and Mullet, J.E. (1993) Plastid genes encoding the transcription/translation apparatus are differentially transcribed early in barley (Hordeum vulgare) chloroplast development (evidence for selective stabilization of psbA mRNA). Plant Physiol. 101, 781-791.

Birch-Machin, I., Newell, C.A., Hibberd, J.M. and Gray, J.C. (2004) Accumulation of rotavirus VP6 protein in chloroplasts of transplastomic tobacco is limited by protein stability. Plant Biotechnol. J. 2, 261-270.

Bock, R. (2007) Plastid biotechnology: prospects for herbicide and insect resistance, metabolic engineering and molecular farming. Curr. Opin. Biotechnol. 18, 100-106.

Brar, B.K., Stephanou, A., Liao, Z., O'Leary, R.M., Pennica, D., Yellon, D.M. and Latchman, D.S. (2001) Cardiotrophin-1 can protect cardiac myocytes from injury when added both prior to simulated ischaemia and at reoxygenation. Cardiovasc Res. 51, 265-274.

Bustos, M., Beraza, N., Lasarte, J.J., Baixeras, E., Alzuguren, P., Bordet, T. and Prieto, J. (2003) Protection against liver damage by cardiotrophin-1: a hepatocyte survival factor up-regulated in the regenerating liver in rats. Gastroenterology, 125, 192-201.

Daniell, H. (1997) Transformation and foreign gene expression in plants by microprojectile bombardment. Methods Mol. Biol. 62, 463-489. 
Daniell, H. (2006) Production of biopharmaceuticals and vaccines in plants via the chloroplast genome. Biotechnol. J. 1, 10711079.

De Cosa, B., Moar, W., Lee, S.B., Miller, M. and Daniell, H. (2001) Overexpression of the Bt cry2Aa2 operon in chloroplasts leads to formation of insecticidal crystals. Nat. Biotechnol. 19, 71-74.

Dhingra, A., Portis, A.R. Jr and Daniell, H. (2004) Enhanced translation of a chloroplast-expressed $R b c S$ gene restores small subunit levels and photosynthesis in nuclear RbcS antisense plants. Proc. Natl. Acad. Sci. USA, 101, 6315-6320.

DuBell, A.N. and Mullet, J.E. (1995) Differential transcription of pea chloroplast genes during light-induced leaf development (continuous far-red light activates chloroplast transcription). Plant Physiol. 109, 105-112.

Eibl, C., Zou, Z., Beck, A., Kim, M., Mullet, J. and Koop, H.U. (1999) In vivo analysis of plastid $p s b A, r b c L$ and $r p / 32$ UTR elements by chloroplast transformation: tobacco plastid gene expression is controlled by modulation of transcript levels and translation efficiency. Plant J. 19, 333-345.

Farran, I., Sanchez-Serrano, J.J., Medina, J.F., Prieto, J. and MingoCastel, A.M. (2002) Targeted expression of human serum albumin to potato tubers. Transgenic Res. 11, 337-346.

Fernandez-San Millan, A., Mingo-Castel, A., Miller, M. and Daniell, H. (2003) A chloroplast transgenic approach to hyper-express and purify human serum albumin, a protein highly susceptible to proteolytic degradation. Plant Biotechnol. J. 1, 71-79.

Fernandez-San Millan, A., Farran, I., Molina, A., Mingo-Castel, A.M. and Veramendi, J. (2007) Expression of recombinant proteins lacking methionine as $\mathrm{N}$-terminal amino acid in plastids: human serum albumin as a case study. J. Biotechnol. 127, 593-604.

Fernandez-San Millan, A., Ortigosa, S.M., Hervas-Stubbs, S., CorralMartínez, P., Seguí-Simarro, J.M., Gaétan, J., Coursaget, P. and Veramendi, J. (2008) Human papillomavirus L1 protein expressed in tobacco chloroplasts self-assembles into virus-like particles that are highly immunogenic. Plant Biotechnol. J. in press.

Fischer, R., Stoger, E., Schillberg, S., Christou, P. and Twyman, R.M. (2004) Plant-based production of biopharmaceuticals. Curr. Opin. Plant Biol. 7, 152-158.

Hagemann, R. (2004) The sexual inheritance of plant organelles. In: Molecular Biology and Biotechnology of Plant Organelles (Daniell, H. and Chase, C.D., eds), pp. 93-114. Dordrecht: Springer.

Herz, S., Fussl, M., Steiger, S. and Koop, H.U. (2005) Development of novel types of plastid transformation vectors and evaluation of factors controlling expression. Transgenic Res. 14, 969-982.

In̄iguez, M., Berasain, C., Martinez-Anso, E., Bustos, M., Fortes, P., Pennica, D., Avila, M.A. and Prieto, J. (2006) Cardiotrophin-1 defends the liver against ischemia-reperfusion injury and mediates the protective effect of ischemic preconditioning. J. Exp. Med. 203, 2809-2815.

Kim, M., Christopher, D.A. and Mullet, J.E. (1993) Direct evidence for selective modulation of $p s b A, r p o A, r b c L$ and 165 RNA stability during barley chloroplast development. Plant Mol. Biol. 22, 447463.

Kozai, T. (2005) Closed system with lamps for high quality transplant production at low costs using minimum resources. In: Photoautotrophic (Sugar-Free Medium) Micropropagation as a New Micropropagation and Transplant Production System (Kozai, T., Afreen, F. and Zobayed, S.M.A., eds), pp. 275-311. Dordrecht: Springer.

Kumar, S., Dhingra, A. and Daniell, H. (2004) Stable transformation of the cotton plastid genome and maternal inheritance of transgenes. Plant Mol. Biol. 56, 203-216.

Kuroda, H. and Maliga, P. (2001) Sequences downstream of the translation initiation codon are important determinants of translation efficiency in chloroplasts. Plant Physiol. 125, 430-436.

Leelavathi, S. and Reddy, V.S. (2003) Chloroplast expression of His-tagged GUS-fusions: a general strategy to overproduce and purify foreign proteins using transplastomic plants as bioreactors. Mol. Breed. 11, 49-58.

Lesbordes, J.C., Cifuentes-Diaz, C., Miroglio, A., Joshi, V., Bordet, T., Kahn, A. and Melki, J. (2003) Therapeutic benefits of cardiotrophin-1 gene transfer in a mouse model of spinal muscular atrophy. Hum. Mol. Genet. 12, 1233-1239.

Lutz, K.A. and Maliga, P. (2007) Construction of marker-free transplastomic plants. Curr. Opin. Biotechnol. 18, 107-114.

Ma, J.K., Chikwamba, R., Sparrow, P., Fischer, R., Mahoney, R. and Twyman, R.M. (2005) Plant-derived pharmaceuticals - the road forward. Trends Plant Sci. 10, 580-585.

Magee, A.M., Horvath, E.M. and Kavanagh, T.A. (2004) Pre-screening plastid transgene expression cassettes in Escherichia coli may be unreliable as a predictor of expression levels in chloroplasttransformed plants. Plant Sci. 166, 1605-1611.

Maliga, P. (2003) Progress towards commercialization of plastid transformation technology. Trends Biotechnol. 21, 20-28.

Maliga, P. (2004) Plastid transformation in higher plants. Annu. Rev. Plant Biol. 55, 289-313.

Marques, J.M., Belza, I., Holtmann, B., Pennica, D., Prieto, J. and Bustos, M. (2007) Cardiotrophin-1 is an essential factor in the natural defense of the liver against apoptosis. Hepatology, 45, 639-648.

Molina, A., Hervas-Stubbs, S., Daniell, H., Mingo-Castel, A.M. and Veramendi, J. (2004) High-yield expression of a viral peptide animal vaccine in transgenic tobacco chloroplasts. Plant Biotechnol. J. 2, 141-153.

Pennica, D., King, K.L., Shaw, K.J., Luis, E., Rullamas, J., Luoh, S.M., Darbonne, W.C., Knutzon, D.S., Yen, R., Chien, K.R., Baker, J.B. and Wood, W.I. (1995) Expression cloning of cardiotrophin 1, a cytokine that induces cardiac myocyte hypertrophy. Proc. Natl. Acad. Sci. USA, 92, 1142-1146.

Pennica, D., Wood, W.I. and Chien, K.R. (1996a) Cardiotrophin-1: a multifunctional cytokine that signals via LIF receptor-gp 130 dependent pathways. Cytokine Growth Factor Rev. 7, 81-91.

Pennica, D., Swanson, T.A., Shaw, K.J., Kuang, W.J., Gray, C.L., Beatty, B.G. and Wood, W.I. (1996b) Human cardiotrophin-1: protein and gene structure, biological and binding activities, and chromosomal localization. Cytokine, 8, 183-189.

Quesada-Vargas, T., Ruiz, O.N. and Daniell, H. (2005) Characterization of heterologous multigene operons in transgenic chloroplasts: transcription, processing, and translation. Plant Physiol. 138, 1746-1762

Reddy, A.R. and Raghavendra, A.S. (2006) Photooxidative stress. In: Physiology and Molecular Biology of Stress Tolerance in Plants (Rao, K.V.M., Raghavendra, A.S. and Reddy, K.J., eds), pp. 157186. Dordrecht, Netherlands: Springer.

Robledo, O., Fourcin, M., Chevalier, S., Guillet, C., Auguste, P., Pouplard-Barthelaix, A., Pennica, D. and Gascan, H. (1997) Signaling of the cardiotrophin-1 receptor. Evidence for a third receptor component. J. Biol. Chem. 272, 4855-4863.

Ruf, S., Karcher, D. and Bock, R. (2007) Determining the transgene containment level provided by chloroplast transformation. Proc. Natl. Acad. Sci. USA, 104, 6998-7002. 
Ruhlman, T., Ahangari, R., Devine, A., Samsam, M. and Daniell, H. (2007) Expression of cholera toxin B-proinsulin fusion protein in lettuce and tobacco chloroplasts - oral administration protects against development of insulitis in non-obese diabetic mice. Plant Biotechnol. J. 5, 495-510.

Sakamoto, W. (2006) Protein degradation machineries in plastids. Annu. Rev. Plant Biol. 57, 599-621.

Staub, J.M. and Maliga, P. (1994) Translation of psbA mRNA is regulated by light via the $5^{\prime}$-untranslated region in tobacco plastids. Plant J. 6, 547-553.

Staub, J.M., Garcia, B., Graves, J., Hajdukiewicz, P.T., Hunter, P., Nehra, N., Paradkar, V., Schlittler, M., Carroll, J.A., Spatola, L., Ward, D., Ye, G. and Russell, D.A. (2000) High-yield production of a human therapeutic protein in tobacco chloroplasts. Nat. Biotechnol. 18, 333-338.

Stevens, L.H., Stoopen, G.M., Elbers, I.J., Molthoff, J.W., Bakker, H.A., Lommen, A., Bosch, D. and Jordi, W. (2000) Effect of climate conditions and plant developmental stage on the stability of antibodies expressed in transgenic tobacco. Plant Physiol. 124, 173-182.

Svab, Z. and Maliga, P. (2007) Exceptional transmission of plastids and mitochondria from the transplastomic pollen parent and its impact on transgene containment. Proc. Natl. Acad. Sci. USA, 104, 7003-7008.

Trebitsh, T., Levitan, A., Sofer, A. and Danon, A. (2000) Translation of chloroplast psbA mRNA is modulated in the light by counteracting oxidizing and reducing activities. Mol. Cell Biol. 20, 11161123.

Tregoning, J.S., Nixon, P., Kuroda, H., Svab, Z., Clare, S., Bowe, F., Fairweather, N., Ytterberg, J., van Wijk, K.J., Dougan, G. and Maliga, P. (2003) Expression of tetanus toxin Fragment $C$ in tobacco chloroplasts. Nucleic Acids Res. 31, 1174-1179.

Watson, J., Koya, V., Leppla, S.H. and Daniell, H. (2004) Expression of Bacillus anthracis protective antigen in transgenic chloroplasts of tobacco, a non-food/feed crop. Vaccine, 22, 4374-4384.

Wen, T.C., Rogido, M.R., Moore, J.E., Genetta, T., Peng, H. and Sola, A. (2005) Cardiotrophin-1 protects cortical neuronal cells against free radical-induced injuries in vitro. Neurosci. Lett. 387, 38-42.

Wirth, S., Segretin, M.E., Mentaberry, A. and Bravo-Almonacid, F. (2006) Accumulation of hEGF and hEGF-fusion proteins in chloroplast-transformed tobacco plants is higher in the dark than in the light. J. Biotechnol. 125, 159-172.

Ye, G.N., Hajdukiewicz, P.T., Broyles, D., Rodriguez, D., Xu, C.W., Nehra,
N. and Staub, J.M. (2001) Plastid-expressed 5-enolpyruvylshikimate-3-phosphate synthase genes provide high level glyphosate tolerance in tobacco. Plant J. 25, 261-270.

Yukawa, M., Kuroda, H. and Sugiura, M. (2007) A new in vitro translation system for non-radioactive assay from tobacco chloroplasts: effect of pre-mRNA processing on translation in vitro. Plant J. 49, 367-376.

Zheng, B., Halperin, T., Hruskova-Heidingsfeldova, O., Adam, Z. and Clarke, A.K. (2002) Characterization of chloroplast Clp proteins in Arabidopsis: localization, tissue specificity and stress responses. Physiol. Plant. 114, 92-101.

Zoschke, R., Liere, K. and Borner, T. (2007) From seedling to mature plant: Arabidopsis plastidial genome copy number, RNA accumulation and transcription are differentially regulated during leaf development. Plant J. 50, 710-722.

Zou, Z., Eibl, C. and Koop, H.U. (2003) The stem-loop region of the tobacco psbA 5'UTR is an important determinant of mRNA stability and translation efficiency. Mol. Genet. Genomics, 269 340-349.

\section{Supplementary material}

The following supplementary material is available for this article:

Figure S1 Homoplasmic plants expressing recombinant human cardiotrophin-1 (rhCT-1) under the control of the psbA promoter and the G10 leader sequence.

This material is available as part of the online article from: http://www.blackwell-synergy.com/doi/abs/10.1111/ j.1467-7652.2008.00334.x

(This link will take you to the article abstract).

Please note: Blackwell Publishing is not responsible for the content or functionality of any supplementary materials supplied by the authors. Any queries (other than missing material) should be directed to the corresponding author for the article. 